\title{
MR Imaging in Corpus Neoplasia: Spectrum of MR Findings
}

\author{
Takashi Koyama • Kaori Togashi
}

Published online: 30 December 2012

(C) Springer Science+Business Media New York 2012

\begin{abstract}
MR imaging is a noninvasive and a useful modality for depicting a wide variety of uterine tumors with excellent tissue contrast. In case malignant uterine neoplasms are suspected, contrast-enhanced image is useful to delineate the tumor extent and internal architecture of the tumor. In cases of endometrial carcinomas, dynamic enhanced imaging is utilized for evaluating the depth of myometrial invasion. In differentiating malignant uterine tumors from benign conditions, in addition to filtration into the surrounding tissues, the presence of necrosis that is usually better demonstrated on postcontrast images is important imaging finding. Diffusion-weighted imaging is an emerging technique that depicts tissue contrast based on restricted diffusion of water molecules and demonstrates increased intensity in the malignant tumors. The recognition of typical MR imaging features in a wide variety of endometrial tumors may facilitate differentiation, thus better determining appropriate patient management.
\end{abstract}

Keywords MR imaging · Diffusion-weighted imaging · Endometrial carcinomas · Leiomyomas ·

Endometrial stromal sarcomas $\cdot$ Leiomyosarcomas .

Endometrial stromal sarcomas · Adenosarcomas ·

Carcinosarcomas $\cdot$ Undifferentiated endometrial sarcomas

\footnotetext{
T. Koyama $(\triangle)$

Department of Diagnostic Radiology, Osaka Red Cross Hospital, Fudegasaki-cho 5-30, Tennoji-ward,

Osaka-city 543-8555, Japan

e-mail: montpeti@kuhp.kyoto-u.ac.jp

\section{K. Togashi}

Department of Diagnostic Radiology, Graduate school

of Medicine, Kyoto University, Kawahara-cho 54, Sakyo-ward, Kyoto-city 606-8507, Japan

e-mail: ktogashi@kuhp.kyoto-u.ac.jp
}

\section{Introduction}

MR imaging is a noninvasive and a useful modality for depicting a wide variety of uterine tumors with excellent tissue contrast. Although transvaginal ultrasonography (TVUS) is widely accepted as the initial modality of choice for evaluating patients with abnormal genital bleeding or infertility, MR imaging can serve as the problem-solving modality when the tissue characterization of the uterine lesion is difficult on TVUS. The characteristics of MR imaging results in several advantages: 1) the evaluation of tumor extent; 2) the demonstration of the internal architecture of the mass, such as cyst formation, hemorrhage, and necrosis; and 3) the determination of tumor origin, i.e., whether it arises from the endometrium, submucosal region, or myometrium.

In case of endometrial carcinomas, MR imaging is usually performed after establishment of the diagnosis. Thereafter, the main role of MR imaging is to evaluate local extension of the tumor, especially with respect to myometrial invasion, cervical invasion, and the presence of extrauterine disease. When the endometrial carcinomas are suspected, the combined use of gadolinium-based contrast media is preferred, because the depth of myometrial invasion can be well evaluated in dynamic enhanced MR image obtained by bolus injection of the contrast material.

In case of myometrial lesions, the diagnosis of the tumors frequently depends on MR findings, because these lesions cannot be approached by hysteroscopic biopsies. In myometrial lesion, MR imaging also is expected to differentiate benign and malignant myometrial tumors. When malignant myometrial tumor is suspected, contrast-enhanced images is useful for differential diagnosis and may provide better contrast of the boundary and the internal architecture of the tumor. In differentiating malignant uterine tumors from benign conditions, in addition to filtration into the surrounding tissues, the presence of necrosis that is usually better demonstrated on postcontrast images is important imaging finding. 
Fig. 1 Endometrial polyp in a 52-year-old woman, who is taking Tamoxifen for her pervious breast cancer. a Sagittal T2weighted image shows a welldefined polypoid tumor of high intensity (arrowheads), containing fine reticular network of low intensity. b Postcontrast T1-weighted image shows unenhanced areas, representing cystic spaces (arrows) a

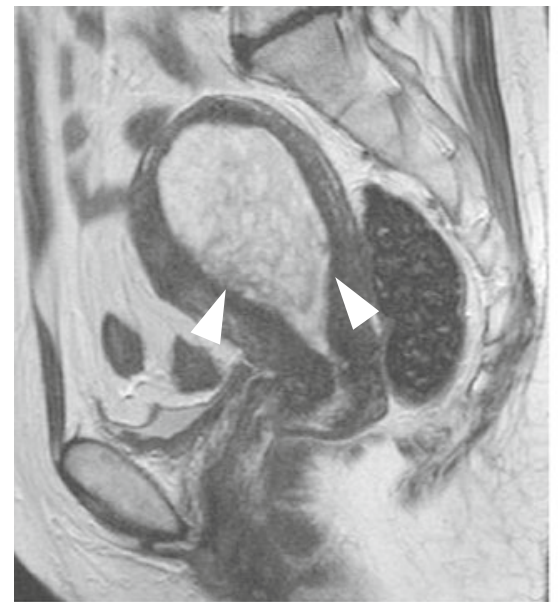

b

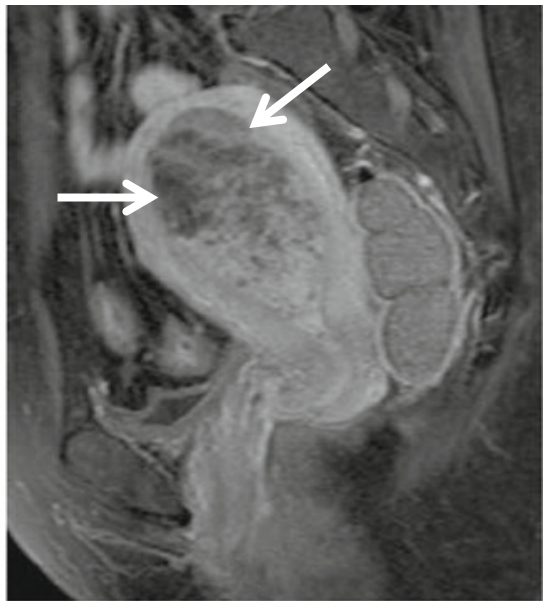

One of the recent topics in MR imaging is the application of diffusion-weighted imaging (DWI) in the female pelvic region [1•]. DWI is an emerging technique used to show tissue characteristics based on diffusion motion of water molecules that is known as Brownian motion [2]. The utility of this technique has been initially established in the central nervous system, mainly for the diagnosis of acute stroke [3, 4]. With the recent advent of fast MR imaging techniques, DWI of the abdomen has become possible with short acquisition times [5]. One of the greatest advantages of DW imaging in the abdomen is that this technique delineates lesions of increased cellularity, which are represented by malignant tumors, as in a region of increased signal intensity with excellent tissue contrast [6-8]. DWI also can provide quantitative measurement of apparent diffusion coefficient (ADC) value, which is considered to be influenced by nuclear-to-cytoplasm ratio and cellular density in solid tissues [9-11]. In uterine tumors, carcinomas and sarcomas are demonstrated as increased intensity, whereas normal endometrium and benign myometrial tumors have decreased intensity [12-14].
The purpose of this article is to revisit the utility of MR imaging by reviewing a spectrum of MR imaging findings in representative corpus tumors. In this review, uterine tumors are roughly divided into benign and malignant, endometrial, and myometrial tumors. Although endometrial stromal sarcomas and undifferentiated stromal sarcomas are considered as endometrial origin, findings in these tumors are described in malignant myometrial tumors, because these tumors may show extensive myometrial invasion or present as myometrial tumor.

\section{Benign Endometrial Tumors}

\section{Endometrial Polyps}

Endometrial polyps represent the most common uterine endometrial pathologic finding, characterized by localized overgrowth of endometrial glandular and stromal tissue covered by epithelium. Polyps may originate anywhere in
Fig. 2 Adenomyoma in a 40year-old woman. a Sagittal T2weighted image demonstrates a polypoid mass of decreased intensity in the endometrium (arrows), containing cystic space of bright intensity. The myometrium in the anterior wall and fundus is diffusely thickened by simultaneous adenomyosis. b T1-weighted image shows high intensity corresponding to the cystic space, suggesting the hemorrhagic fluid within the mass a

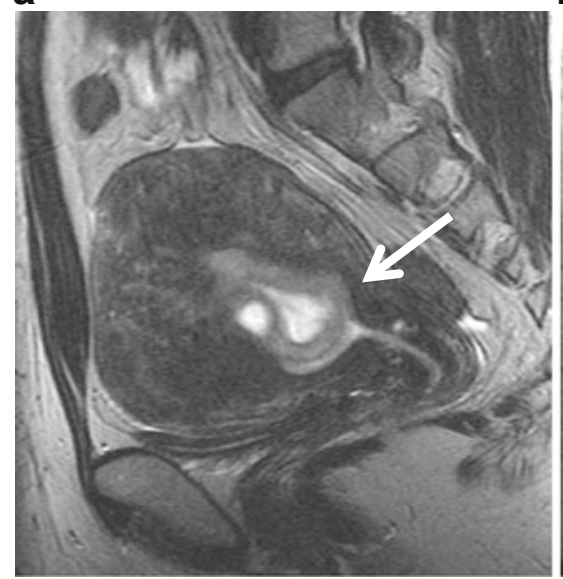

b

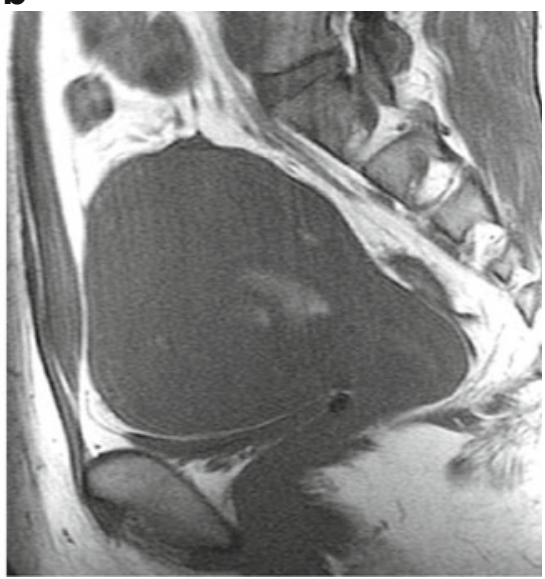


Fig. 3 Endometrioid

carcinoma in a 30 -year-old woman. a Sagittal T2-weighted image demonstrates an illdefined area of decreased intensity in the thickened endometrium (arrows). b Postcontrast T1-weighted image demonstrates poorly enhanced tumor in the endometrium (arrows). In both images, the endometrialmyometrial border is well preserved, suggesting the tumor is confined within the endometrium a

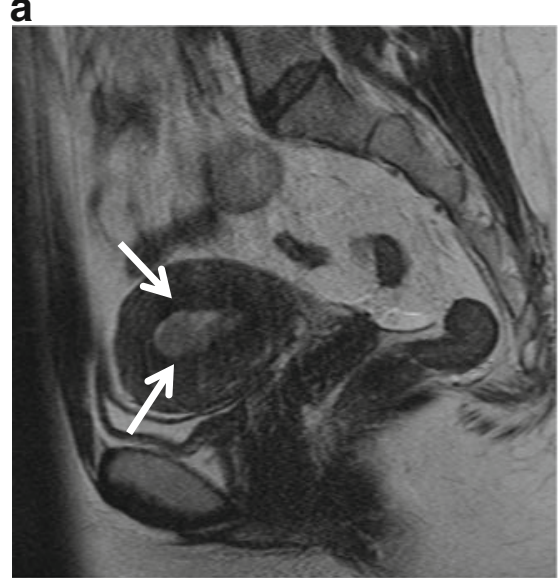

b

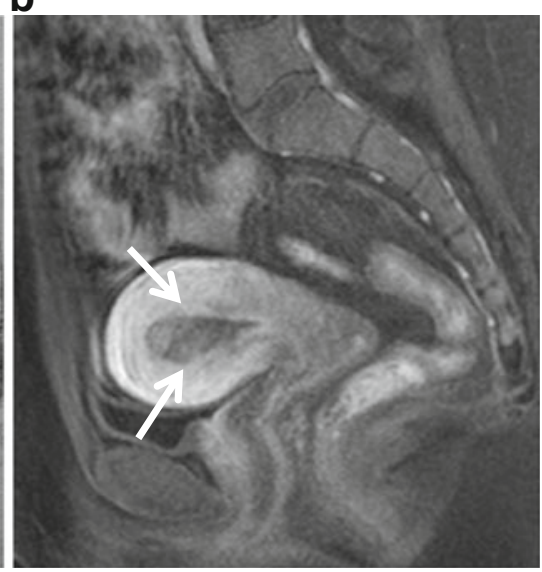

the uterine cavity, but most occur in the fundus, particularly in the cornal region. Endometrial polyps consist of variable amounts of smooth-muscle fibers within the stroma. Endometrial polyps may occur with or without endometrial hyperplasia, but endometrial polyps are a more common cause of endometrial thickening than endometrial hyperplasia.

Typical MR features of endometrial polyps include a central fibrous core of low signal intensity and multiple intratumoral cysts of high signal intensity on T2-weighted images (Fig. 1) [15]. Otherwise, endometrial polyps can be considered to represent mere endometrial thickening. The stalks of endometrial polyps are not usually apparent on MR imaging, as fluid does not outline the polyps. Endometrial polyps are more frequently seen in women taking Tamoxifen for breast carcinoma [16]. Endometrial polyps associated with Tamoxifen are usually large (mean diameter $5 \mathrm{~cm}$ ) and cystic glandular dilatation and stromal proliferation can be prominent [17].

\section{Adenomyoma/Adenofibromas}

When the stroma is predominantly composed of smooth muscle fibers or fibrous stroma, the lesions are designated as polypoid adenomyomas and adenofibroma, respectively. Polypoid adenomyomas accounts for approximately $2 \%$ of all endometrial polyps and are typically seen in premenopausal women. Atypical polypoid adenomyoma (APA) is a rare variant of a polypoid adenomyoma, microscopically characterized by architectural and cytologic atypia. These tumors most frequently present as pedunculated or sessile polypoid masses in the lower uterine segment or endocervix [18]. On MR imaging, the lesion typically presents as a hypointense polypoid mass [19, 20]. Adenomyoma may contain cystic space of bright intensity, resulting from menstrual hemorrhage, and is called cystic adenomyoma (Fig. 2) [21•].
Fig. 4 Endometrioid carcinoma in 53-year-old woman. a Sagittal T2-weighted image demonstrates an ill-defined endometrial tumor. The endometrial-myometrial border is almost entirely obscured, suggesting the myometrial invasion by the tumor. b Earlyphase image of dynamic enhanced T1-weighted clearly demonstrates inner myometrium of strong enhancement (arrowheads), which become irregular by invading tumor

\section{a}

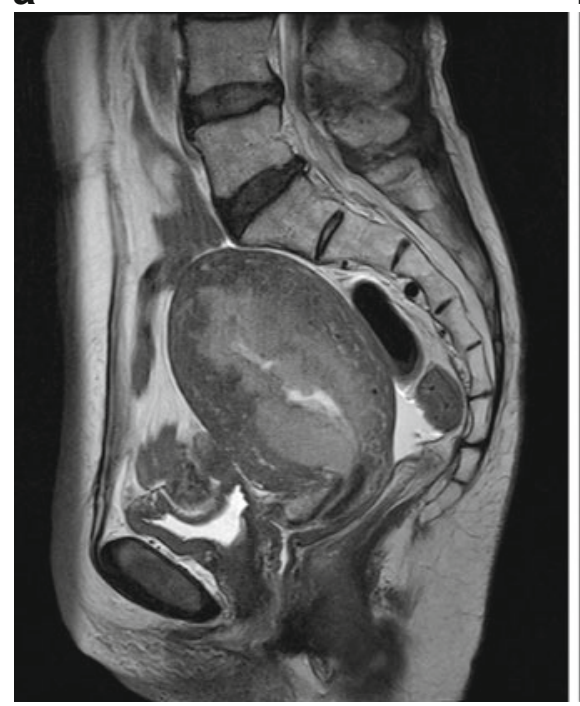

b

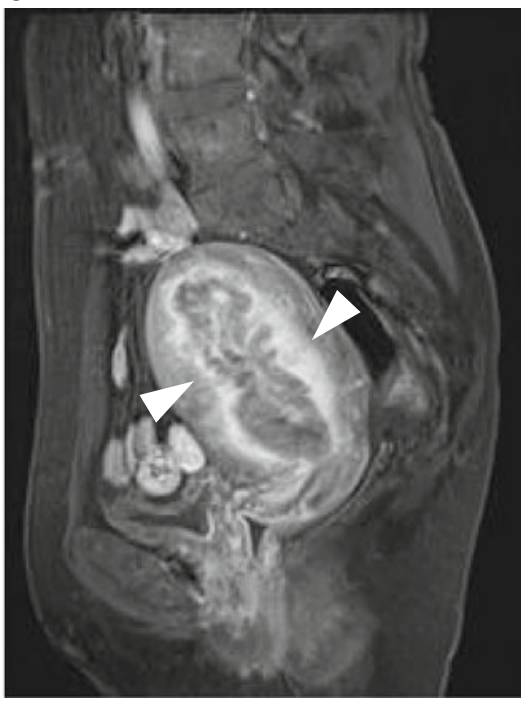




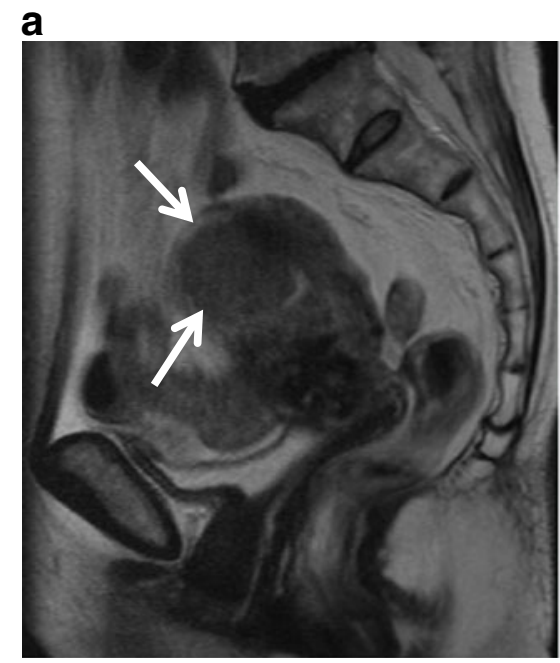

Fig. 5 Serous carcinoma in a 55-year-old female. a Sagittal T2-weighted image demonstrates an endometrial tumor of decreased intensity occupying the endometrial cavity, and protruding into the external cervical os. Note the endometrial-myometrial border in both the corpus and cervix is diffusely obscured, suggesting myometrial invasion of the tumor

\section{Malignant Endometrial Tumors}

\section{Endometrial Carcinomas}

Endometrial carcinoma is the most common gynecologic malignancy; $95 \%$ of endometrial carcinomas occur in women who are older than age 40 years [22]. MR imaging findings of endometrial carcinomas can vary from a polypoid endometrial mass to an endophytic tumor infiltrating into the myometrium. The tumors are depicted as foci of either low or high signal intensity on T2-weighted images and of decreased enhancement than the surrounding myometrium on contrast-enhanced, T1-weighted images (Fig. 3). Because MR imaging is usually performed after establishment of the diagnosis in patients with b

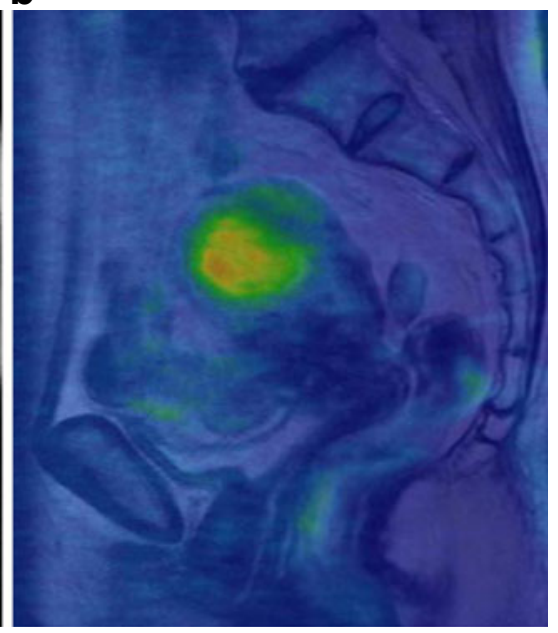

(arrowheads). There is a small implant on the posterior serosal surface of the uterus (arrow). b Fusion image of DWI onto T2-weighted image exhibits increased intensity corresponding to the tumor in the endometrial cavity. The implantation on the posterior wall is also clearly demonstrated with excellent tissue contrast (arrowhead)

endometrial carcinoma, the main role of MR imaging is local staging, especially with respect to myometrial invasion, cervical invasion, and extrauterine disease. The myometrial invasion is suspected by recognition of the disruption or irregularity of the inner myometrium that exhibits distinct low intensity on T2-weighted images, and early enhancement on dynamic contrast MR imaging (Fig. 4). Cervical stromal invasion is suggested when the stroma of low intensity become disrupted or irregular by the tumor (Fig. 5). Haldorsen et al. has reported that MR imaging is superior to endocervical curettage in preoperative assessment of cervical stromal invasion [23•]. DWI also demonstrates foci of carcinoma as an area of increased intensity. This emerging technique is useful to demonstrate the extent of the tumor, especially in case myometrial invasion is

Fig. 6 Endometrioid carcinoma in 69-year-old woman. a T2-weighted image demonstrates an ill-defined myometrial mass of decreased intensity (arrows). b Fusion image with DWI onto T2weighted image shows increased intensity in the myometrial mass, which represents endometrioid carcinoma infiltrating into the myometrium

\section{a}

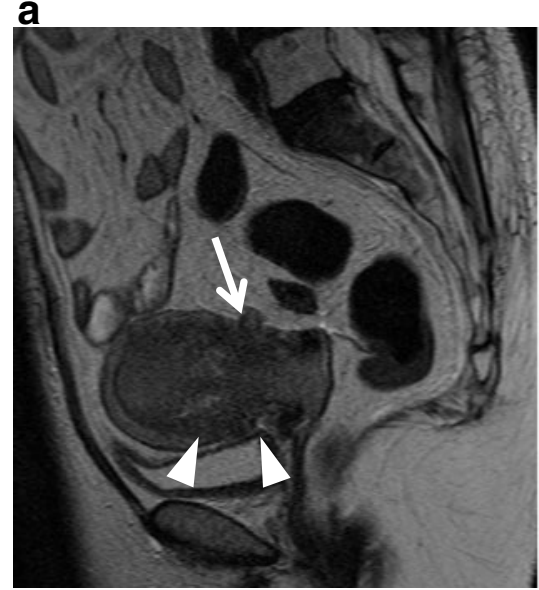

b

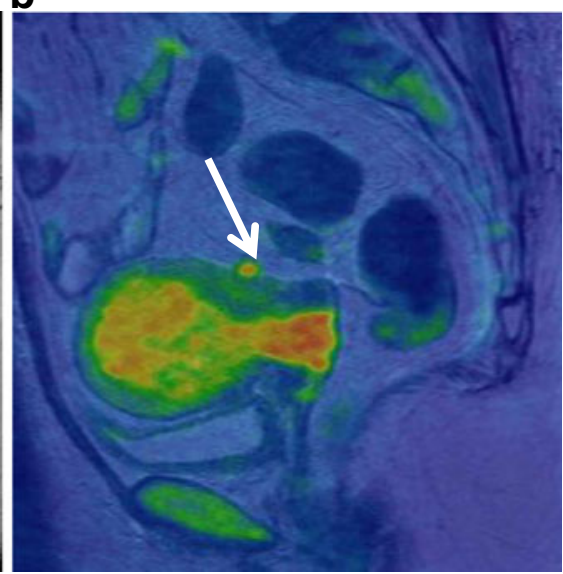


Fig. 7 Small cell carcinoma in a 40-year-old woman. a Sagittal T2-weighted image demonstrates a polypoid mass of heterogeneous intensity in the endometrial cavity. The myometrium is diffusely thickened by diffusely infiltrating tumor. The cervix also is involved by the tumor (arrowhead). Extensive lymphadenopathy in the para-aortic region represents nodal metastases (arrows). b Sagittal contrast-enhanced T1weighted images shows multifocal, unenhanced areas of necrosis a

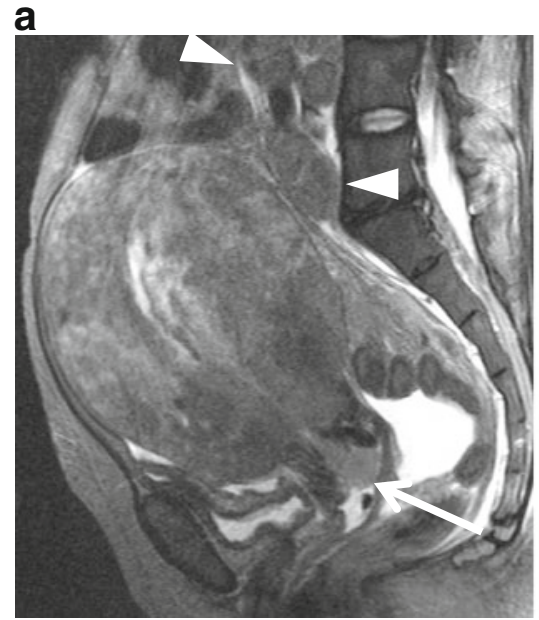

b

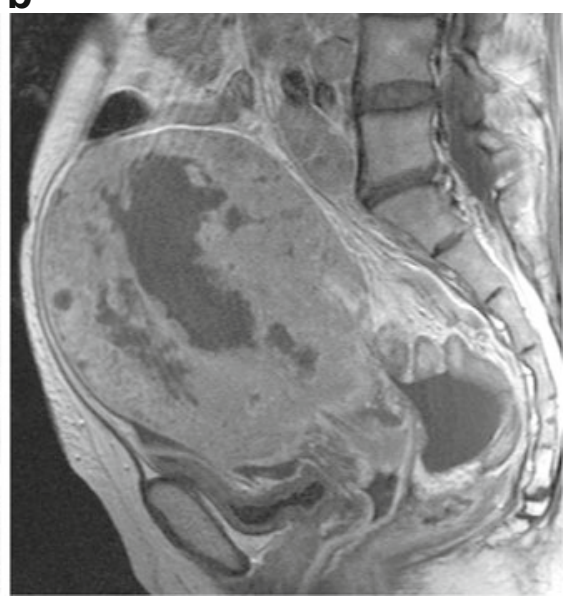

obscure on conventional images (Fig. 6). In recent reports, fused DWI and T2-weighted image is better than dynamic contrast-enhanced MR imaging [24•,25•]. The ADC measured in DWI also correlates with histologic grade of the endometrioid carcinomas [13]. In 2009, the FIGO staging system for endometrial cancer was significantly revised; key changes include simplification of stage I disease and removal of cervical mucosal invasion as a distinct stage $[26 \bullet, 27 \bullet]$. These changes has important implications for radiologists, because MR imaging will provide better diagnostic accuracies in newly revised staging and, consequently, will play more important role in preoperative assessment of the endometrial tumors [28•].

The majority of endometrial carcinomas are represented by endometrial carcinoma. Other histologic subtypes of endometrial carcinoma include serous carcinomas, clear cell carcinomas, and small cell carcinomas. These tumors are more frequently associated with deep myometrial invasion, nodal metastases, and a worse prognosis compared with high-grade endometrioid carcinomas [29-32]. On MR imaging, these carcinomas tend to be exophytic and may occur within an endometrial polyp. In small cell carcinoma, MR imaging may show diffuse and extensive myometrial invasion (Fig. 7) [33].

\section{Adenosarcomas}

Adenosarcomas are characterized by benign epithelium and mesenchymal components, which are typically composed of endometrial stromal sarcomatous tissue. Adenosarcomas are commonly large polypoid tumors with an average diameter of $5 \mathrm{~cm}$. Approximately $10 \%$ of adenosarcomas may be associated with sarcomatous overgrowth, in which pure sarcomatous components account for $25 \%$ or more of the tumor [34]. Such tumors frequently invade into the myometrium and have a correspondingly worse prognosis. MR imaging features of adenosarcomas can vary from a bulky polypoid mass to multiple polypoid masses of decreased signal intensity on T2weighted imaging (Fig. 8) [35]. The tumor may contain high-
Fig. 8 Adenosarcoma in a 27year-old woman. a Sagittal T2weighted image shows adenosarcoma composed of numerous polypoid mass, which protrude into the endometrial cavity. Multiple punctate foci of high intensity within the tumor represent microscopically dilated glands in the tumor. The disruption of the endometrialmyometrial border and heterogeneously increased intensity in the anterior wall represents invasion of the tumor into the myometrium (arrows). b Postcontrast T1-weighted image shows good enhancement in the tumor a

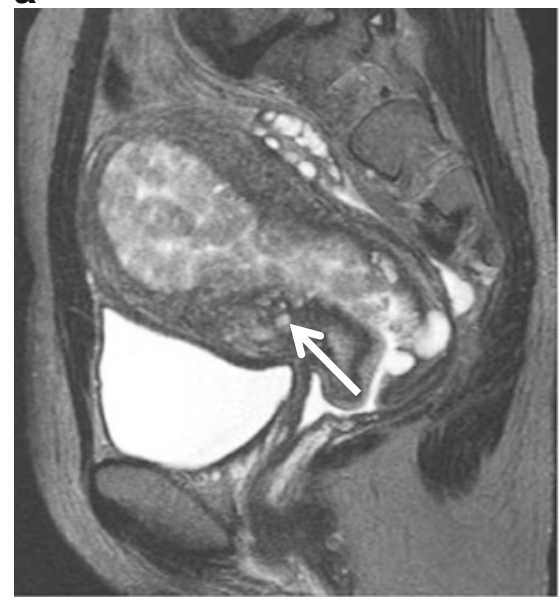

b

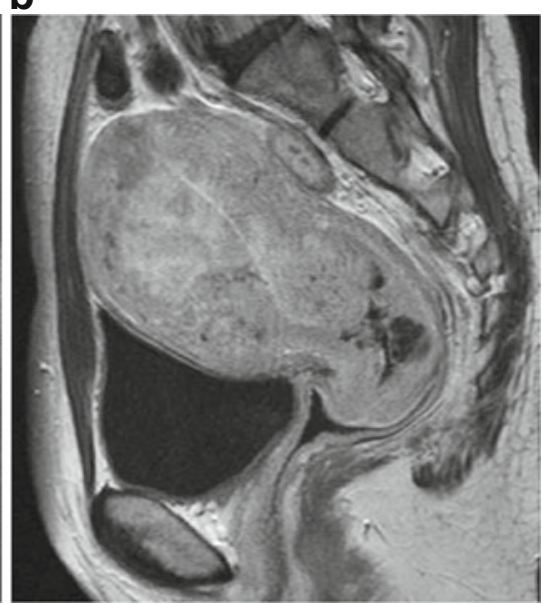




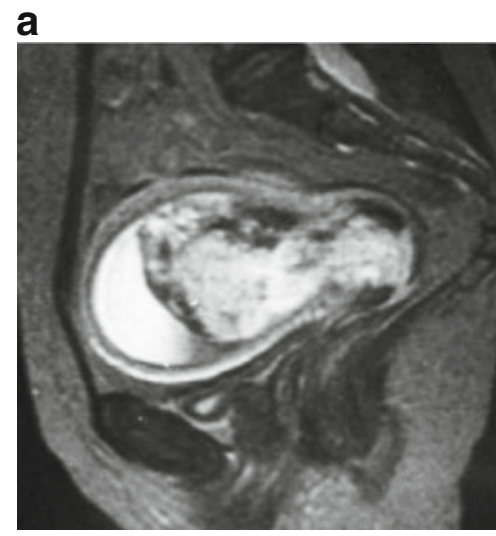

b

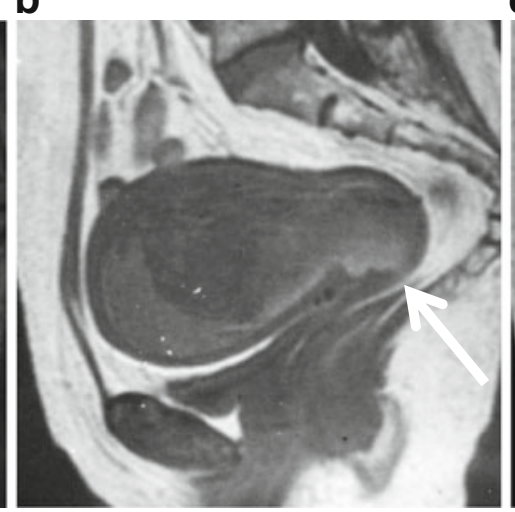

C

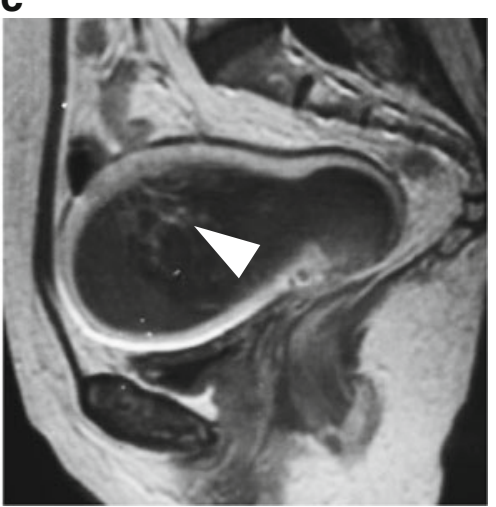

Fig. 9 Carcinosarcoma in a 66-year-old female. a T2-weighted image demonstrates a large polypoid tumor (arrowheads) of heterogeneously high intensity, containing fresh hemorrhage of distinct low intensity. The tumor protrudes into the cervical canal. Fluid-fluid level in the uterine cavity represents hematometra. b T1-weighted image shows area of high intensity (arrow) in the tumor, suggesting intratumoral hemorrhage. c Postcontrast T1-weighted image shows fine reticular enhancement in the lesion (arrowheads) intensity foci on T2-weighted images, which are considered to represent dilated epithelial glands.

\section{Carcinosarcomas}

Carcinosarcomas account for approximately $2 \%$ of all uterine malignancies and typically affect elderly postmenopausal women [36]. Histologically, carcinosarcomas are characterized by the presence of both epithelial and mesenchymal malignancies. Recent clinicopathologic immunohistochemical and molecular studies have provided compelling evidence that carcinosarcomas should be classified as high-grade variants of endometrioid carcinoma.

On MR imaging, carcinosarcoma typically shows a bulky polypoid mass that protrudes into the cervical canal. The tumor typically exhibits low intensity on T1-weighted images and heterogeneously high signal intensity on T2-weighted imaging [37, 38], associated with foci of extensive hemorrhage and necrosis within the tumor, which show high signal intensity on T1-weighted images, low signal intensity on T2-weighted images, and a lack of enhancement on contrast-enhanced images (Fig. 9) [39].

\section{Benign Myometrial Tumors}

\section{Adenomyosis}

Adenomyosis typically present as either diffuse or focal thickening of the inner myometrium or an ill-defined myometrial mass of low intensity on T2-weighted images (Fig. 2). The ectopic endometrial tissue within adenomyosis may be depicted as foci of increased intensity (Fig. 10). These foci may contain hemorrhage of high intensity on T1-weighted images. Occasionally, these ectopic endometrial glands may contain hemorrhage resulting from menstrual bleeding, depicted as foci of
Fig. 10 Adenomyosis in a 40year-old woman. a T2-weighted image shows ill-defined low intensity in the diffusely thickened anterior myometrial wall. b T1weighted image shows hemorrhage in the ectopic endometrial tissue as foci of high intensity

\section{a}

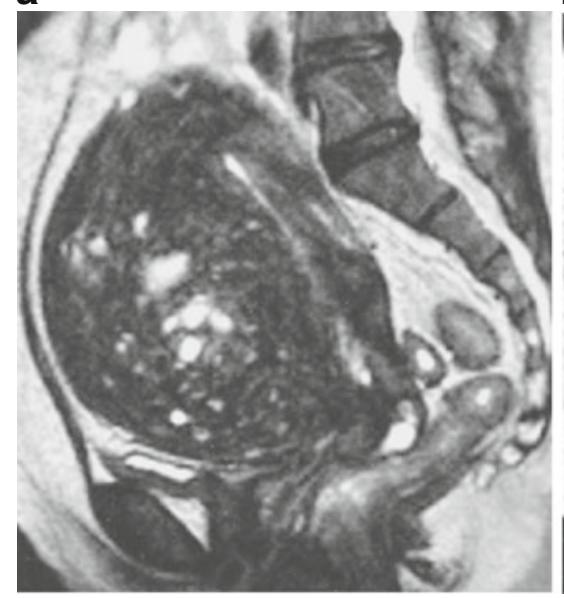

b

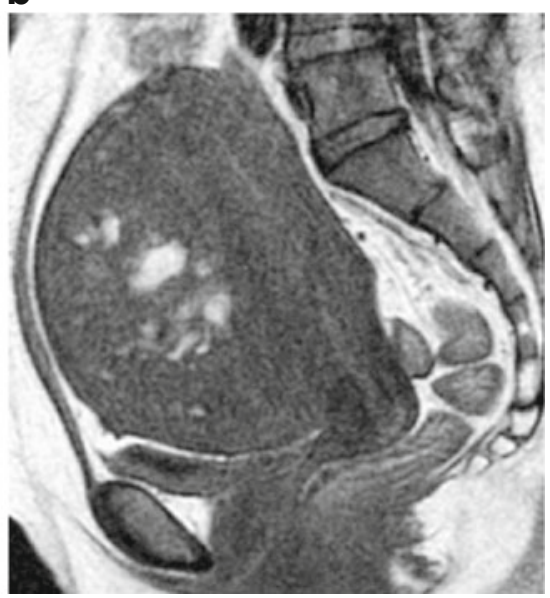


high intensity on T1-weighted images $[21 \bullet, 40]$. When these cystic foci become prominent, the lesion is called as adenomyotic cyst. Adenomyotic cyst can be submucosal, intramyometrial, or subserosal.

\section{Leiomyomas}

Leiomyomas are the most common uterine tumor, histologically characterized by the benign proliferation of smooth muscles cell separated by variable amounts of collagenous stroma. MR imaging is the most accurate modality for detecting and diagnosing leiomyomas. Leiomyoma typically presents as a well-defined myometrial mass of distinct low intensity on T2-weighted images, reflecting abundant collagenous stroma (Fig. 11) [41]. Leiomyomas may show variable MR appearance due to degenerations and unusual growth patterns. Clinically, large subserosal leiomyomas are frequently mistaken as adnexal mass. In those instances, MR imaging is useful in diagnosing the origin of the mass by clearly demonstrating the tumor vessels extending from the myometrium to the tumor, known as bridgingvascular sign (Fig. 12).

Leiomyoma with edema may show heterogeneously increased intensity on T2-weighted images. The typical speckled appearance on T2-weighted images may help to diagnose this type of degenerated leiomyoma [42]. These leiomyomas usually do not contain foci of hemorrhage or necrosis. Cellular leiomyoma is one of the histologic variants characterized by increased cellularity and also shows increased intensity on T2-weighted images. Homogeneous appearance of the tumor favors benign nature of the lesion.

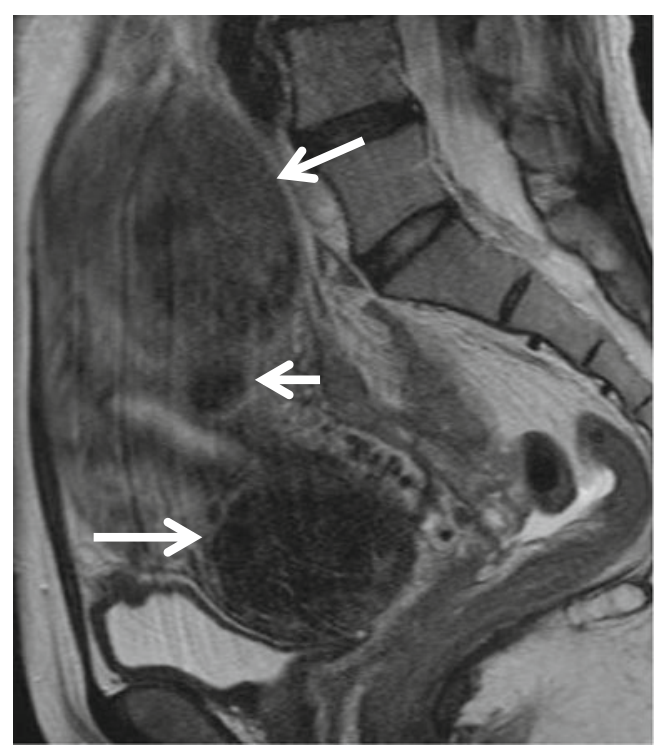

Fig. 11 Leiomyoma in a 42-year-old woman. Sagittal T2-weighted image demonstrates well-demarcated masses of distinct low intensity in the myometrium (arrows)

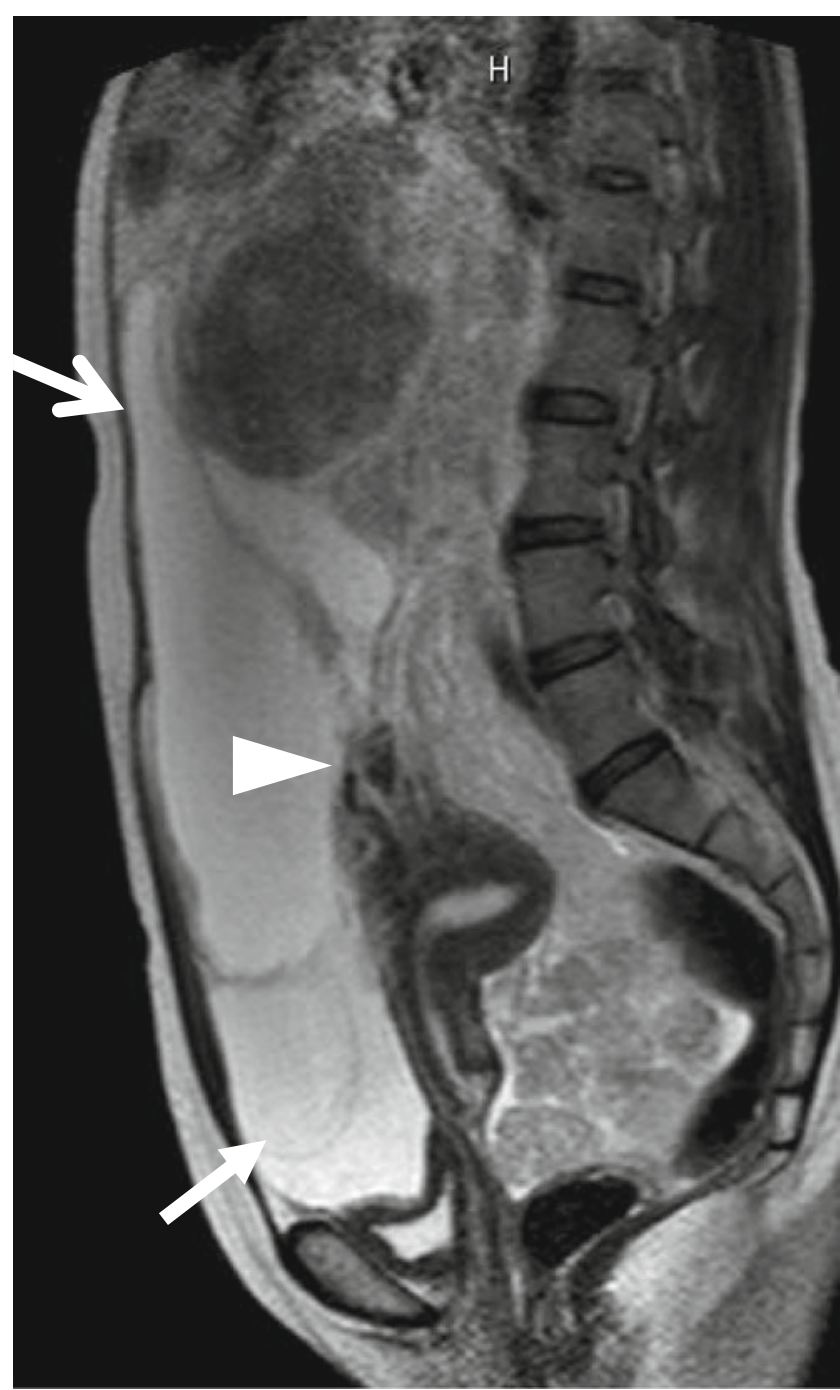

Fig. 12 A huge subserosal leiomyoma in a 40-year-old woman. a Sagittal T2-weighted image demonstrates a huge heterogenous tumor consisting of cystic components of bright intensity anteriorly (arrows) and solid component of intermediate to low intensity. Note the tumor vessels extending from the myometrium to the tumor (arrowhead)

Leiomyoma with red degeneration may contain extensive hemorrhage, which exhibit increased intensity on T1weighted images, sometimes associated with thick rim of low signal intensity on T2-weighted images (Fig. 13). Leiomyoma with red degeneration is well circumscribed and do not invade into the adjacent tissues. This peculiar degeneration is considered to be result from hemorrhagic infarction caused by obstruction of drainage veins and typically occurs in young females during pregnancy or from taking oral contraceptives [43].

Benign uterine leiomyomas may show unusual growth pattern that should not be mistaken for malignancy [42, 44]. Leiomyomas may show extensive venous extension, which is known as intravenous leiomyomatosis. Subserosal uterine leiomyoma extending into the broad ligament or the 
Fig. 13 Red degeneration in a 36-year-old woman. a Sagittal T2-weighted image demonstrates a well-defined myometrial mass of low intensity, associated with thick rim of distinct low signal intensity (arrows). b T1-weighted image reveals entirely increased intensity in the tumor, suggesting red degeneration

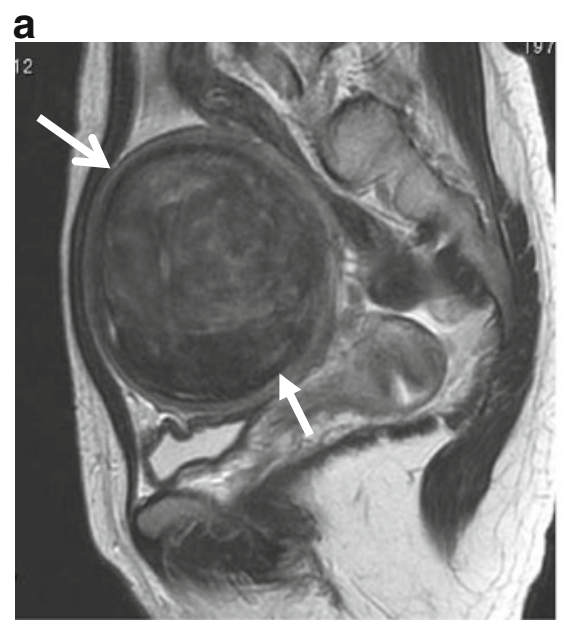

b

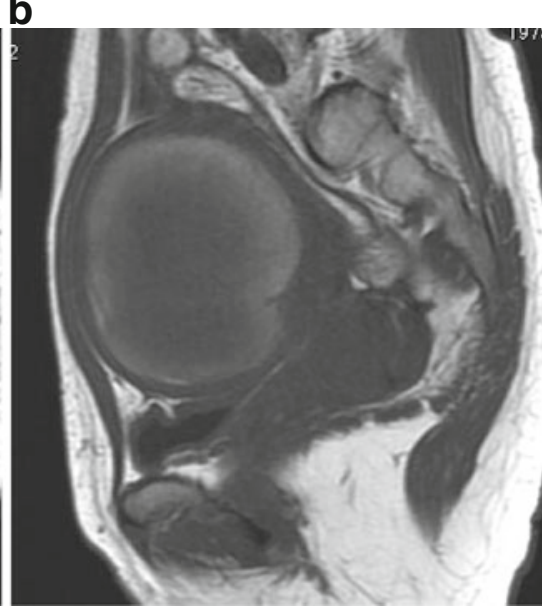

retroperitoneum also may produce infiltrative appearance. Rarely, submucosal myomas can be pedunculated and prolapse into the cervical canal or vagina [45].

\section{Malignant Myometrial Tumors}

\section{Leiomyosarcoma}

Leiomyosarcoma represents the most common uterine sarcoma, characterized by tumor cells demonstrating smooth muscle differentiation, associated with prominent coagulative necrosis and marked nuclear atypia. Although the tumors commonly occur in postmenopausal women with a median age of 50-55 years, $15 \%$ of patients are younger than age 40 years [46]. The prognosis is generally poor, with 5 -year survival rates of $15-25 \%$ [47]. On MR imaging, leiomyosarcoma presents as an ill-defined tumor mainly located within the myometrium and exhibiting low signal intensity on T1-weighted images and intermediate to high signal intensity on T2-weighted images (Fig. 14). The foci of intratumoral hemorrhage or necrosis exhibit focal high intensity on T1-weighted images and decreased intensity on T2-weighted images. DWI also is a useful technique that demonstrates sarcomas as increased intensity [14].

\section{Endometrial Stromal Sarcoma}

Endometrial stromal sarcoma (ESS) is the second most common uterine sarcoma and is characterized by proliferation of well-differentiated cells closely resembling cells of endometrial stroma in the proliferative phase of the menstrual cycle. ESS tends to occur in a young age group (mean age 39 years) [48]. Curettage may not be helpful to establish diagnosis, because the cells shed from ESS usually are not sufficiently atypical to be distinguished from benign stromal cells. Nonetheless, despite scant cytologic atypia, ESS tends
Fig. 14 Leiomyosarcoma in a 60 -year-old woman. a Sagittal T2-weighted image demonstrates an irregular-shaped large mass of heterogeneous intensity in the posterior wall of the uterus (arrows). b Postcontrast T1-weighted images with fat suppression demonstrate multifocal, unenhanced areas of necrosis within the tumor a

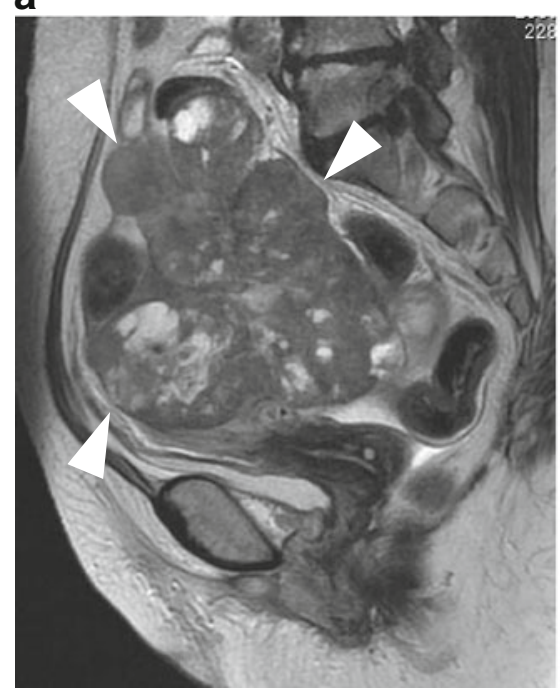

b

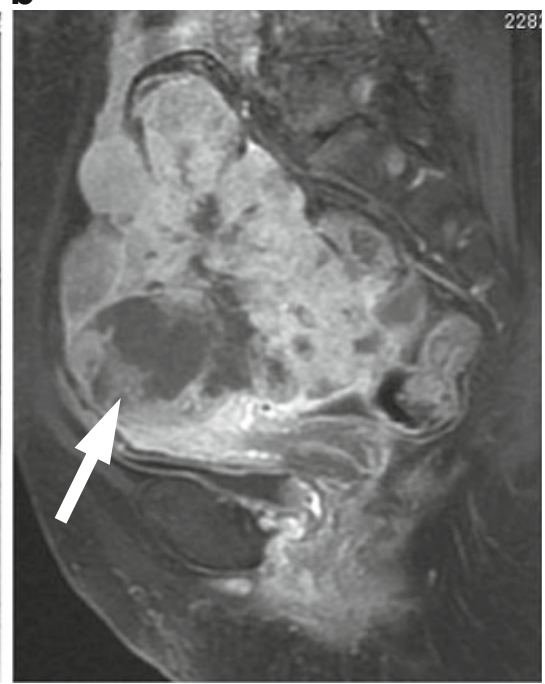


Fig. 15 Endometrial stromal sarcoma in a 47-year-old woman. a Sagittal T2-weighted image demonstrates an ill-defined lesion of slightly increased intensity in the posterior myometrium of the uterus (arrows). Note the bands of low signal intensity within the lesion, which represent preserved myometrial bundles amidst the infiltrating nests of tumors cells. b Fusion image with DWI onto T2weighted image reveals increased signal corresponding to the infiltration tumor in the myometrium a

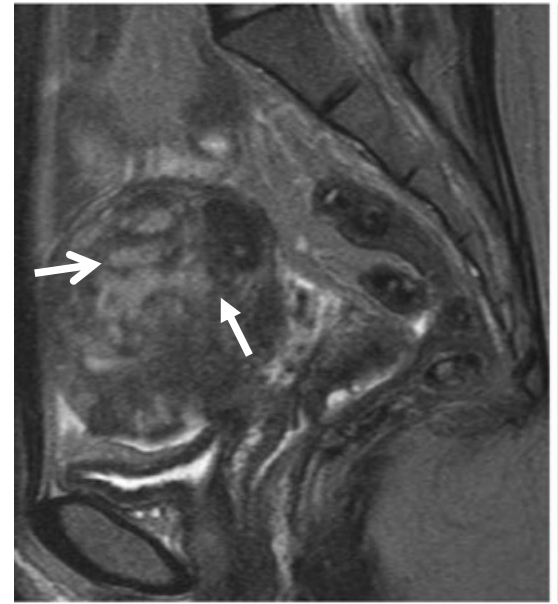

b

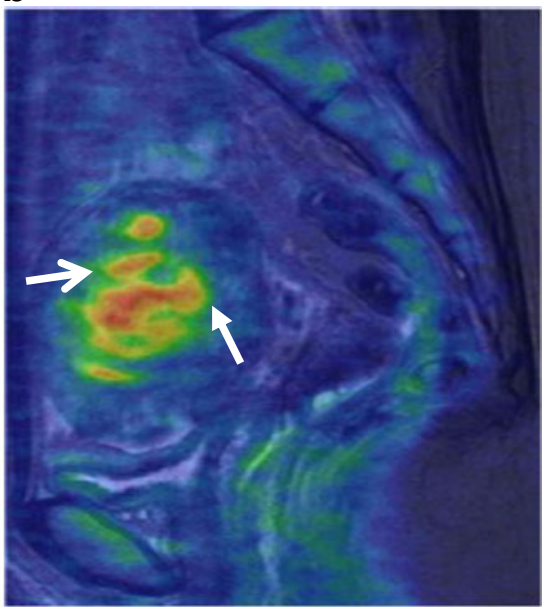

to invade into the myometrium and surrounding structures. When the tumor is advanced, the 5-year survival rate is unfavorable (40-50 \%) [49].

Although MR features of ESS are variable, ranging from a polypoid mass confined within the endometrial cavity to a tumor with an extensive myometrial invasion, commonly the tumor is entirely within the myometrium. The tumors typically exhibit increased signal intensity on $\mathrm{T} 2$-weighted imaging. One of the characteristic MR findings is the presence of bands of low signal intensity within the myometrial lesion, which represent preserved myometrial bundles amidst the infiltrating nests of tumors cells (Fig. 15) [48, 49]. As well as leiomyosarcomas, the nest of ESS is depicted as areas of increased intensity on DWI [14]. Hemorrhage and necrosis are unusual in this tumor. Occasionally, the tumor may extend into the broad ligament or adnexa, as well as demonstrate extensive intravenous involvement [48].

\section{Undifferentiated Endometrial Sarcoma}

Undifferentiated endometrial sarcoma is a highly aggressive tumor, characterized by highly anaplastic cells that lack specific differentiation [47, 50], typically affecting postmenopausal women with a mean age of 61 years at diagnosis. Conventionally, these tumors have been classified as high-grade ESS. Recently, some of these tumors have been considered to be variants of carcinosarcoma without carcinomatous component. Occasionally, undifferentiated endometrial sarcoma may present as an infiltrative mass entirely within the myometrium. Most patients die of tumor dissemination within 3 years. MR features

\section{a}

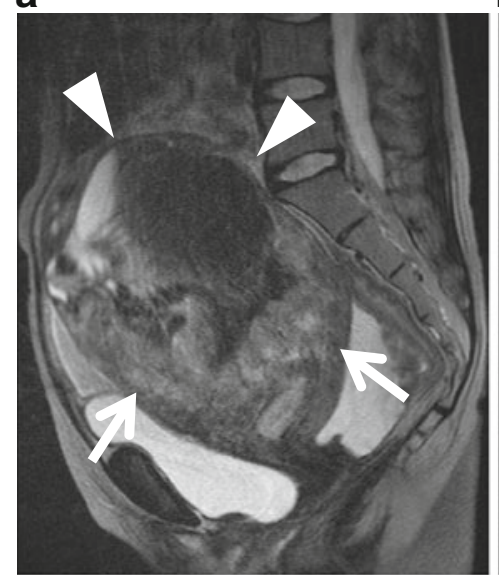

b

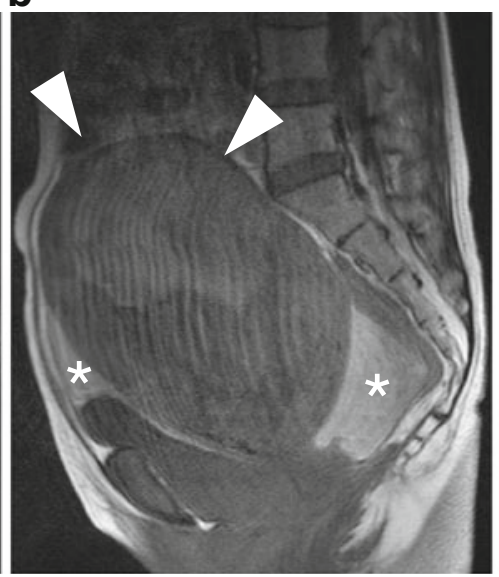

c

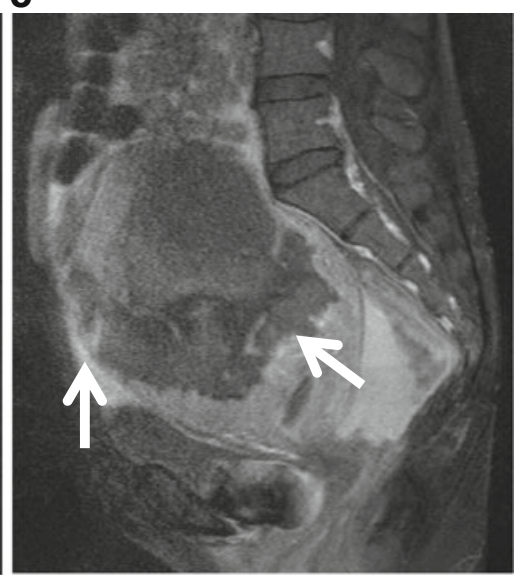

Fig. 16 Undifferentiated stromal sarcoma in a 22-year-old female who presented with acute abdomen due to uterine rupture. a Sagittal T2weighted image demonstrates an ill-defined myometrial mass of heterogeneous appearance in the anterior wall (arrows). The upper portion of the tumor containing fluid-fluid level represents hemorrhagic necrosis within the tumor (arrowheads). b T1-weighted image demonstrates increased signal intensity corresponding to the area of hemorrhagic necrosis (arrowheads). Hemorrhagic ascites of increased signal intensity also is present in the Douglas fossa and uterovesical fossa (asterisks). c Postcontrast T1-weighted image with fat suppression demonstrates heterogeneous enhancement within the solid component of the tumor (arrows) 
of undifferentiated endometrial sarcoma are similar to those in leiomyosarcomas or carcinomasarcomas, characterized by heterogeneous appearance with foci of increased intensity on T1weighted and low signal intensity on T2-weighted images, reflecting hemorrhage and necrosis within the tumor (Fig. 16).

\section{Conclusions}

MR imaging is a useful modality for depicting a wide variety of uterine tumors with excellent tissue contrast. When malignant uterine neoplasms are suspected, gadolinium-based contrast material is useful in delineating the tumor extent and internal architecture of the tumor. In addition to conventional technique, DWI also is useful to demonstrate lesion of increased cellularity, represented by malignant tumors. Benign endometrial tumors, such as endometrial polyps, adenofibromas, and adenomyomas, are characterized as polypoid lesions, occasionally associated with multiple intratumoral cysts of high intensity on T2-weighted images. MR imaging findings of endometrial carcinomas can vary from a polypoid endometrial mass to an endophytic tumor infiltrating into the myometrium, of either low or high intensity on T2-weighted images, decreased enhancement than the surrounding myometrium on contrast enhanced T1-weighted images. Both adenosarcomas and carcinosarcomas are typically large polypoid masses, and the latter typically contains extensive hemorrhage and necrosis. Leiomyomas are well-defined masses of low intensity. Endometrial stromal sarcomas are typically infiltrating myometrial mass, preserving the myometrial bundles of low signal intensity within the tumor. Leiomyosarcomas and undifferentiated stromal sarcoma are heterogeneous mass containing hemorrhage and necrosis. The recognition of typical MR imaging features in a wide variety of endometrial tumors may facilitate differentiation, thus better determining appropriate patient management.

Disclosures No conflicts of interest relevant to this article were reported.

\section{References}

Papers of particular interest, published recently, have been highlighted as:

- Of importance

1. - Punwani S. Diffusion weighted imaging of female pelvic cancers: concepts and clinical applications. Eur J Radiol. 2011;78(1):21-9. This introduces state of the art diffusion-weighted imaging for pelvic cancer.

2. Le Bihan D, Turner R, Douek P, Patronas N. Diffusion MR imaging: clinical applications. AJR Am J Roentgenol. 1992;159 (3):591-9.
3. Bahn MM, Oser AB, Cross 3rd DT. CT and MRI of stroke. J Magn Reson Imaging. 1996;6(5):833-45.

4. Li TQ, Takahashi AM, Hindmarsh T, Moseley ME. ADC mapping by means of a single-shot spiral MRI technique with application in acute cerebral ischemia. Magn Reson Med. 1999;41(1):143-7.

5. Thoeny HC, De Keyzer F. Extracranial applications of diffusionweighted magnetic resonance imaging. Eur Radiol. 2007;17 (6):1385-93.

6. Ichikawa T, Haradome H, Hachiya J, Nitatori T, Araki T. Diffusion-weighted MR imaging with a single-shot echoplanar sequence: detection and characterization of focal hepatic lesions. AJR Am J Roentgenol. 1998;170(2):397-402.

7. Takahara T, Imai Y, Yamashita T, Yasuda S, Nasu S, Van Cauteren M. Diffusion weighted whole body imaging with background body signal suppression (DWIBS): technical improvement using free breathing, STIR and high resolution 3D display. Radiat Med. 2004;22(4):275-82.

8. Marini C, Iacconi C, Giannelli M, Cilotti A, Moretti M, Bartolozzi C. Quantitative diffusion-weighted MR imaging in the differential diagnosis of breast lesion. Eur Radiol. 2007;21(10):2646-55.

9. Lyng H, Haraldseth O, Rofstad EK. Measurement of cell density and necrotic fraction in human melanoma xenografts by diffusion weighted magnetic resonance imaging. Magn Reson Med. 2000;43(6):828-36.

10. Nonomura $\mathrm{Y}$, Yasumoto $\mathrm{M}$, Yoshimura R, Haraguchi $\mathrm{K}$, Ito $\mathrm{S}$, Akashi T, et al. Relationship between bone marrow cellularity and apparent diffusion coefficient. J Magn Reson Imaging. 2001;13 (5):757-60.

11. Szafer A, Zhong J, Gore JC. Theoretical model for water diffusion in tissues. Magn Reson Med. 1995;33(5):697-712.

12. Koyama T, Tamai K, Togashi K. Current status of body MR imaging: fast MR imaging and diffusion-weighted imaging. Int $\mathrm{J}$ Clin Oncol. 2006;11(4):278-85.

13. Tamai K, Koyama T, Saga T, Umeoka S, Mikami Y, Fujii S, et al. Diffusion-weighted MR imaging of uterine endometrial cancer. J Magn Reson Imaging. 2007;26(3):682-7.

14. Tamai K, Koyama T, Saga T, Morisawa N, Fujimoto K, Mikami Y, et al. The utility of diffusion-weighted MR imaging for differentiating uterine sarcomas from benign leiomyomas. Eur Radiol. 2008;18(4):723-30.

15. Grasel RP, Outwater EK, Siegelman ES, Capuzzi D, Parker L, Hussain SM. Endometrial polyps: MR imaging features and distinction from endometrial carcinoma. Radiology. 2000;214(1):47-52.

16. Le Donne M, Lentini M, De Meo L, Benedetto V, Mesiti M. Uterine pathologies in patients undergoing tamoxifen therapy for breast cancer: ultrasonographic, hysteroscopic and histological findings. Eur J Gynaecol Oncol. 2005;26(6):623-6.

17. Ascher SM, Johnson JC, Barnes WA, Bae CJ, Patt RH, Zeman RK. MR imaging appearance of the uterus in postmenopausal women receiving tamoxifen therapy for breast cancer: histopathologic correlation. Radiology. 1996;200(1):105-10.

18. Young RH, Treger T, Scully RE. Atypical polypoid adenomyoma of the uterus. A report of 27 cases. Am J Clin Pathol. 1986;86 (2):139-45.

19. Yamashita Y, Torashima M, Hatanaka Y, Takahashi M, Fukumatsu $\mathrm{K}$, Tanaka N, et al. MR imaging of atypical polypoid adenomyoma. Comput Med Imaging Graph. 1995;19(4):351-5.

20. Haque TL, Togashi K, Koyama T, Fujii S, Konishi J. An unusual presentation of endometrial polyp. Eur Radiol. 2002;12(12):2906-9.

21. - Takeuchi M, Matsuzaki K. Adenomyosis: usual and unusual imaging manifestations, pitfalls, and problem-solving MR imaging techniques. Radiographics. 2011;31(1):99-115. This article is a comprehensive review for adenomyosis, introducing state-of-the art MR imaging techniques.

22. Frei KA, Kinkel K. Staging endometrial cancer: role of magnetic resonance imaging. J Magn Reson Imaging. 2001;13 (6):850-5. 
23. - Haldorsen IS, Berg A, Werner HM, Magnussen IJ, Helland H, Salvesen OO, et al. Magnetic resonance imaging performs better than endocervical curettage for preoperative prediction of cervical stromal invasion in endometrial carcinomas. Gynecol Oncol. 2012;126(3):413-8. This article show clinical practicality of MR imaging by showing its utility in the evaluation of cervical invasion.

24. - Lin G, Ng KK, Chang CJ, Wang JJ, Ho KC, Yen TC, et al. Myometrial invasion in endometrial cancer: diagnostic accuracy of diffusion-weighted 3.0-T MR imaging-initial experience. Radiology. 2009;250(3):784-92. This article demonstrates the superiority of diffusion-weighted imaging to conventinoal MR techniques for staging of the endometrial carcinomas.

25. - Rechichi G, Galimberti S, Signorelli M, Perego P, Valsecchi MG, Sironi S. Myometrial invasion in endometrial cancer: diagnostic performance of diffusion-weighted MR imaging at 1.5-T. Eur Radiol. 2010;20(3):754-62. This article demonstrates the superiority of diffusion-weighted imaging to conventinoal MR techniques for staging of the endometrial carcinomas.

26. - Freeman SJ, Aly AM, Kataoka MY, Addley HC, Reinhold C, Sala E. The revised FIGO staging system for uterine malignancies: implications for MR imaging. Radiographics. 2012;32(6):180527. This clarifies the implications of new FIGO staging systems of endometrial cancer for MR imaging.

27. • Beddy P, O'Neill AC, Yamamoto AK, Addley HC, Reinhold C, Sala E. FIGO staging system for endometrial cancer: added benefits of MR imaging. Radiographics. 2012;32(1):241-54. This clarifies the implications of new FIGO staging systems of endometrial cancer for MR imaging.

28. • Shin KE, Park BK, Kim CK, Bae DS, Song SY, Kim B. MR staging accuracy for endometrial cancer based on the new FIGO stage. Acta Radiol. 2011;52(7):818-24. This article shows that MR can provide improved staging accuracies in the revised FIGO stating system.

29. Hamilton CA, Cheung MK, Osann K, Chen L, Teng NN, Longacre $\mathrm{TA}$, et al. Uterine papillary serous and clear cell carcinomas predict for poorer survival compared to grade 3 endometrioid corpus cancers. Br J Cancer. 2006;94(5):642-6.

30. Carcangiu ML, Chambers JT. Early pathologic stage clear cell carcinoma and uterine papillary serous carcinoma of the endometrium: comparison of clinicopathologic features and survival. Int $\mathbf{J}$ Gynecol Pathol. 1995;14(1):30-8.

31. Sakuragi N, Hareyama H, Todo Y, Yamada H, Yamamoto R, Fujino T, et al. Prognostic significance of serous and clear cell adenocarcinoma in surgically staged endometrial carcinoma. Acta Obstet Gynecol Scand. 2000;79(4):311-6.

32. Eichhorn JH, Young RH. Neuroendocrine tumors of the genital tract. Am J Clin Pathol. 2001;115(Suppl):S94-112.

33. Tamai K, Koyama T, Saga T, Mikami Y, Fujii S, Togashi K. Small cell carcinoma of the uterine corpus: MR imaging and pathological correlation. J Comput Assist Tomogr. 2007;31:485-9.

34. Clement PB. Mullerian adenosarcomas of the uterus with sarcomatous overgrowth. A clinicopathological analysis of 10 cases. Am J Surg Pathol. 1989;13(1):28-38.

35. Lee HK, Kim SH, Cho JY, Yeon KM. Uterine adenofibroma and adenosarcoma: CT and MR findings. J Comput Assist Tomogr. 1998;22(2):314-6.
36. Arrastia CD, Fruchter RG, Clark M, Maiman M, Remy JC, Macasaet $\mathrm{M}$, et al. Uterine carcinosarcomas: incidence and trends in management and survival. Gynecol Oncol. 1997;65(1):158-63.

37. Worthington JL, Balfe DM, Lee JK, Gersell DJ, Heiken JP, Ling D, et al. Uterine neoplasms: MR imaging. Radiology. 1986;159 (3):725-30.

38. Ohguri T, Aoki T, Watanabe H, Nakamura K, Nakata H, Matsuura $\mathrm{Y}$, et al. MRI findings including gadolinium-enhanced dynamic studies of malignant, mixed mesodermal tumors of the uterus: differentiation from endometrial carcinomas. Eur Radiol. 2002;12 (11):2737-42.

39. Bharwani N, Newland A, Tunariu N, Babar S, Sahdev A, Rockall AG, et al. MRI appearances of uterine malignant mixed mullerian tumors. AJR Am J Roentgenol. 2010;195(5):1268-75.

40. Tamai K, Togashi K, Ito T, Morisawa N, Fujiwara T, Koyama T. MR imaging findings of adenomyosis: correlation with histopathologic features and diagnostic pitfalls. Radiographics. 2005;25 (1):21-40.

41. Murase E, Siegelman ES, Outwater EK, Perez-Jaffe LA, Tureck RW. Uterine leiomyomas: histopathologic features, MR imaging findings, differential diagnosis, and treatment. Radiographics. 1999;19(5):1179-97.

42. Ueda H, Togashi K, Konishi I, Kataoka ML, Koyama T, Fujiwara $\mathrm{T}$, et al. Unusual appearances of uterine leiomyomas: MR imaging findings and their histopathologic backgrounds. Radiographics. 1999;19 Spec No:S131-45.

43. Zaloudek C, Norris HJ. Mesenchymal tumors of the uterus. In: Kurman RJ, editor. Blaustein's pathology of the female genital tract. New York: Springer-Verlag; 1994. p. 487-98.

44. Tanaka YO, Nishida M, Tsunoda H, Okamoto Y, Yoshikawa H. Smooth muscle tumors of uncertain malignant potential and leiomyosarcomas of the uterus: MR findings. J Magn Reson Imaging. 2004;20(6):998-1007.

45. Panageas E, Kier R, McCauley TR, McCarthy S. Submucosal uterine leiomyomas: diagnosis of prolapse into the cervix and vagina based on MR imaging. AJR Am J Roentgenol. 1992;159 (3):555-8.

46. Major FJ, Blessing JA, Silverberg SG, Morrow CP, Creasman WT, Currie JL, et al. Prognostic factors in early-stage uterine sarcoma. A Gynecologic Oncology Group study. Cancer. 1993;71(4 Suppl):1702-9.

47. Hendrickson MR. Mesenchymal tumours and related lesions. In: Tavassoli, editor. World Health Organization: tumours and breast and female genital organs. Lyon: IARC press; 2003. p. 233-44.

48. Koyama T, Togashi K, Konishi I, Kobayashi H, Ueda H, Kataoka ML, et al. MR imaging of endometrial stromal sarcoma: correlation with pathologic findings. AJR Am J Roentgenol. 1999;173 (3):767-72.

49. Zaloudek C, Hendrickson MR. Mesenchymal tumors of the uterus. In: Kurman R, editor. Blaustein's pathology of the female genital tract. 5th ed. New York: Springer-Verlag; 2002. p. 561-615.

50. Evans HL. Endometrial stromal sarcoma and poorly differentiated endometrial sarcoma. Cancer. 1982;50(10):2170-82. 